\title{
Diode-pumped polymeric dye lasers operating at a pump power level of $10 \mathrm{~mW}$
}

\author{
Hajime Sakata ${ }^{\text {a) }}$ and Hideki Takeuchi \\ Department of Electrical and Electronic Engineering, Faculty of Engineering, Shizuoka University, \\ Hamamatsu 432-8561, Japan
}

(Received 8 January 2008; accepted 28 February 2008; published online 20 March 2008)

\begin{abstract}
We present a low-threshold vertical-cavity dye laser that is pumped by an InGaN laser diode. This vertical-cavity laser has a $1.2-\mu \mathrm{m}$-thick poly- $N$-vinylcarbazole active layer doped with coumarin 540A. The threshold pump power depended on the dye concentration and showed the minimum value of $16 \mathrm{~mW} /$ pulse with the dye concentration of $4.0 \%$. Single-mode laser action is attained at around $550 \mathrm{~nm}$ and the output intensity decreases to half of the initial value after the emission of $8 \times 10^{5}$ pulses. This concept helps to realize a highly compact dye laser that can be operated in the entire range of visible wavelengths. (C) 2008 American Institute of Physics.
\end{abstract}

[DOI: 10.1063/1.2900628]

There has been considerable interest in organic lasers due to the variety of organic dyes and the flexibility of the device architectures. The broad lasing bands of these devices cover the midvisible wavelengths that cannot be realized by inorganic laser diodes (LDs). The current-injection mode of organic lasers is the most attractive technique for realizing compact organic lasers. On the other hand, considering the durability of dye molecules, optically pumped organic lasers have an advantage in that the active element is separated from the pumping element and, therefore, the former can be easily replaced. Thus far, various types of optically pumped organic lasers have been reported ${ }^{1-3}$ however, the pump sources for such lasers continue to be either the frequency doubled or tripled solid-state lasers or high-power gas lasers, which are expensive, bulky, and consume large amounts of power. In order to reduce the size and energy consumption of the lasers, the pump sources should be replaced with LDs. It is preferable to use violet and blue LDs to excite organic lasers that operate in the visible wavelength range. Recently, waveguide-type and vertical-cavity-type polymeric dye lasers were oscillated using InGaN LDs, which required a threshold pump power of more than $100 \mathrm{~mW} .{ }^{4,5}$ We intend to reduce the pump power required for laser operation to the milliwatt level in order to extend the lifetime of the organic dyes and enable the realization of diode-pumped polymeric dye lasers with low power consumption. In this letter, we report a reduction of the pump LD power that is required for lasing on the basis of a vertical cavity structure.

The diode-pumped polymeric dye laser comprises a pump LD, collimator and focusing lens, and dye laser element (DLE), as shown in Fig. 1. The focusing optics of this laser can be ignored when the DLE is packaged with the pump LD. We used the InGaN-based blue LD (Nichia) as a pump source; the light emitted by this source had a wavelength of $441 \mathrm{~nm}$. We operated the blue LD with a pulsedcurrent circuit that utilized an avalanche transistor to produce an optical pulse with a duration of $\sim 3 \mathrm{~ns}$. The DLE is comprised of a polymeric active layer that is sandwiched between two (top and bottom) distributed Bragg reflectors (DBRs). Such a vertical-cavity laser structure has a large

\footnotetext{
a) Electronic mail: thsakat@ipc.shizuoka.ac.jp.
}

aperture to accept the output light of the pump LDs and it can be arranged in two-dimensional configurations. We doped the active layer with coumarin 540A (C540A, Exciton) since its absorption band matches the emission wavelength of the blue LD. ${ }^{6}$ In order to evaluate the spectroscopic characteristics of the C540A-doped polymeric active layer, we coated glass substrates with poly- $N$-vinylcarbazole (PVK) films by spin coating a cyclohexanone solution of C540A and PVK. Four PVK films were doped with C540A with concentrations of $1.0,2.5,4.0$, and 5.5 wt $\%$. The absorption spectra of the 1 - $\mu$ m-thick C540A-doped PVK films with various dye concentrations were measured by a spectrophotometer (UVmini-1240, Shimadzu) and are shown in Fig. 2. By increasing the dye concentration, it was found that the absorbance curve monotonically increases around the peak wavelength of $430 \mathrm{~nm}$. Based on the absorption spectra, it was observed that the absorption coefficients were in the range of $0.16-0.62 \mu \mathrm{m}^{-1}$ at $441 \mathrm{~nm}$ for dye concentrations between $1.0 \%$ and $5.5 \%$. Figure 2 also shows the measured profiles of the photoluminescence (PL) spectra of the films excited by the blue LD. When the dye concentration was $4.0 \%$ or less, the PL peak remained at approximately $500 \mathrm{~nm}$ due to a small overlap of the absorption band with the PL peak. A further increase in the dye concentration induced a

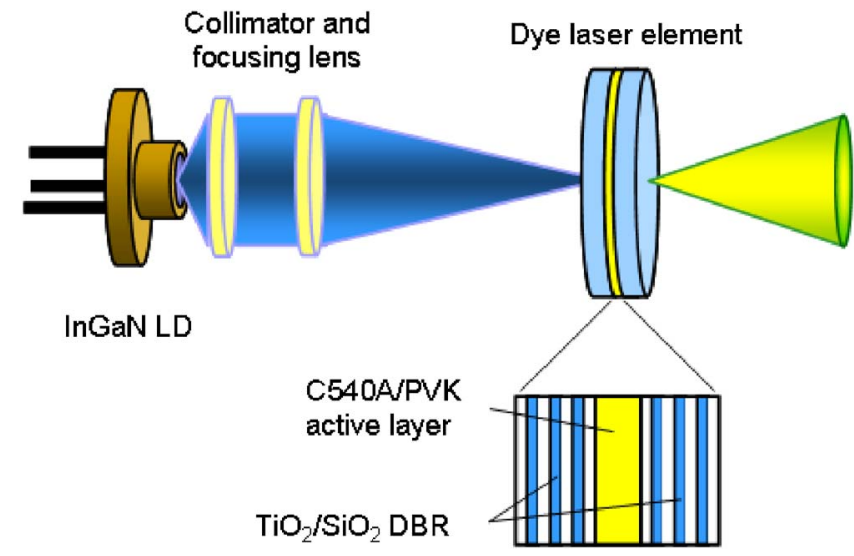

FIG. 1. (Color online) Schematic diagram of the diode-pumped polymeric dye laser consisting of a dye-doped active layer sandwiched between two DBRs. 


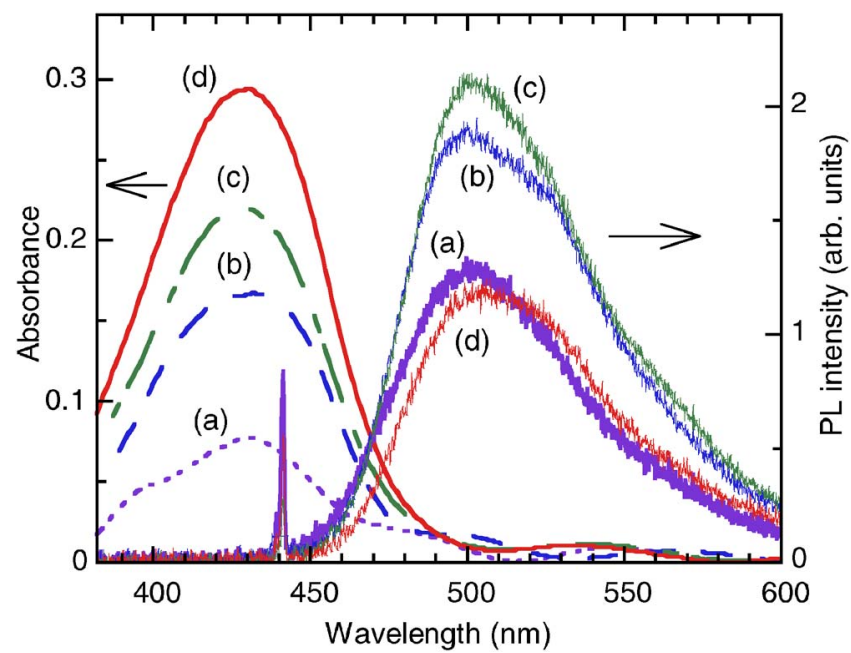

FIG. 2. (Color online) Absorption and PL spectra of the PVK films doped with C540A at various concentrations: (a) $1.0 \%$, (b) $2.5 \%$, (c) $4.0 \%$, and (d) $5.5 \%$.

slight redshift of the PL peak. This result implies that concentration quenching occurs in the 5.5\%-doped sample.

It is preferable to use the microcavity configuration to attain a single longitudinal mode operation in the verticalcavity laser; however, the absorption coefficients derived from Fig. 2 are not sufficient to achieve effective absorption in a one-wave resonant cavity. In order to obtain efficient absorption of the pump LD power and consequently attain an adequate gain-length product, we increased the thickness of the C540A-doped PVK layer within the range where singlemode oscillation could be practically maintained. The DLE was fabricated by sandwiching a C540A-doped PVK layer with a thickness of $1.2 \mu \mathrm{m}$ between the top and bottom DBRs that were fabricated using ten pairs of $\mathrm{TiO}_{2} / \mathrm{SiO}_{2}$ quarter-wave thin films. The reflectance of the DBR was found to be $R=0.994$ at $532 \mathrm{~nm}$. The threshold gain of the vertical cavity is estimated as $g_{\text {th }} \cong-\ln \left(R^{2}\right) / 2 n d=30 \mathrm{~cm}^{-1}$, where $n$ is the refractive index and $d$ is the thickness of the active layer. Such a gain coefficient is attainable with dyedoped polymeric films. ${ }^{7}$ The pump pulse from the blue LD was focused onto the DLE in the form of a circular spot with a diameter of $\sim 20 \mu \mathrm{m}$. The emitted light was monitored using an optical spectrometer (HR4000, Ocean Optics). Figure 3(a) shows the spontaneous emission spectrum of the DLE containing $4.0 \%$ of C540A. The spectrum was modulated by vertical-cavity resonance, thereby enhancing the emission intensity in the three passbands at 503, 551, and $610 \mathrm{~nm}$. One of the stop band edges due to the $\mathrm{TiO}_{2} / \mathrm{SiO}_{2}$ DBR was found at $470 \mathrm{~nm}$, while the other edge at $650 \mathrm{~nm}$ could not be observed because of the lack of spontaneous emission at that wavelength. Figure 3(b) shows the emission spectrum of the DLE when the pump power of the LD was increased to $24 \mathrm{~mW}$. Single-mode lasing was observed at $551 \mathrm{~nm}$ with a spectral width of $0.5 \mathrm{~nm}$ (full width at half maximum). Since the wavelength resolution of the optical spectrometer was $0.25 \mathrm{~nm}$, the spectral width was almost determined by the resolution limit. Single-mode oscillation was achieved by the following mechanism. The passband of $551 \mathrm{~nm}$ was located at the center of the stop band and was separated by approximately $50 \mathrm{~nm}$ from the subsequent passbands. For a short-wavelength passband of $503 \mathrm{~nm}$, the absorption-band edge suppressed the laser emission, while a Downloaded 20 Mar 2008 to 133.70 .80 .52 . Redistribution subject
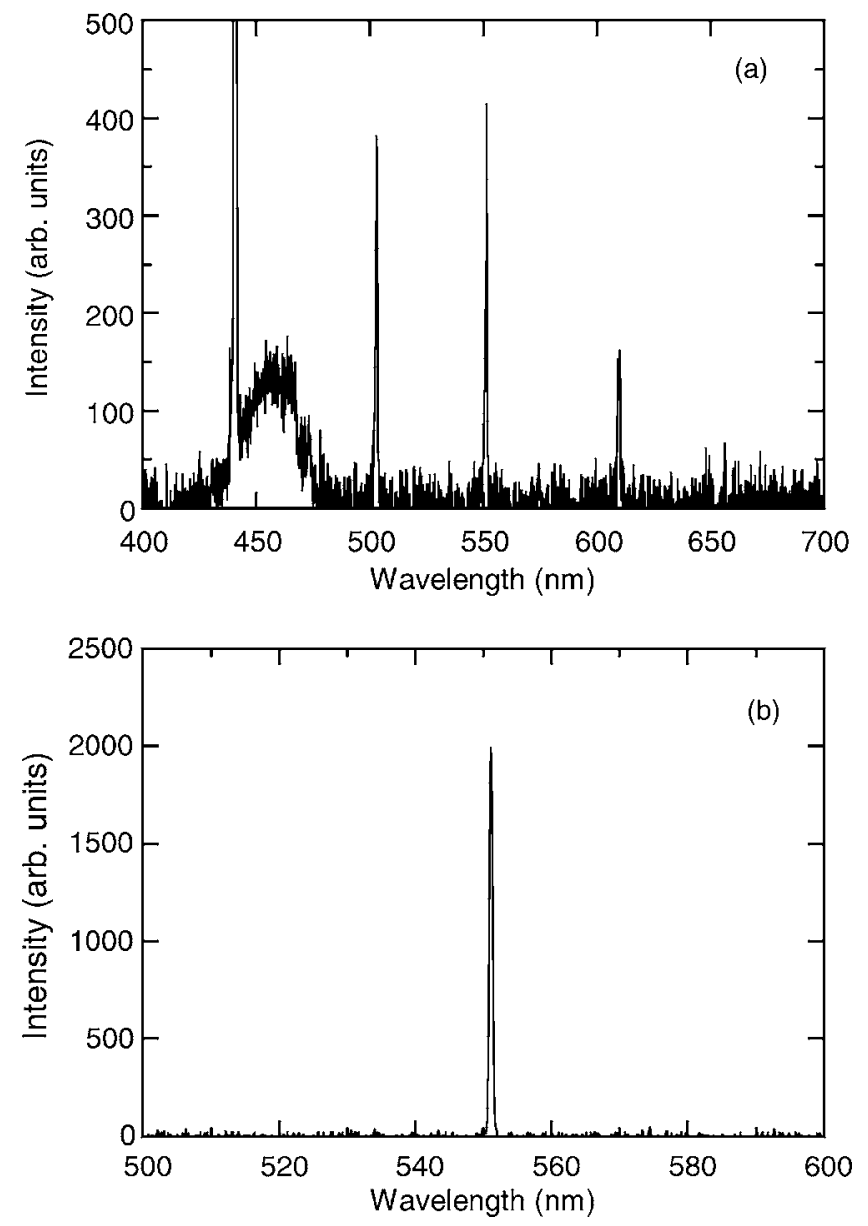

FIG. 3. PL spectra of the DLE pumped by the pulsed blue LD with peak powers of (a) $14 \mathrm{~mW}$ and (b) $24 \mathrm{~mW}$.

long-wavelength passband of $610 \mathrm{~nm}$ was located at the tail of the PL spectrum.

As shown in Fig. 4, we measured the dependence of the emission intensity on the pump pulse power using DLEs with different dye concentrations. Single-mode oscillation was found to occur in all the samples at around $550 \mathrm{~nm}$. As the dye concentration increased, the threshold pump power

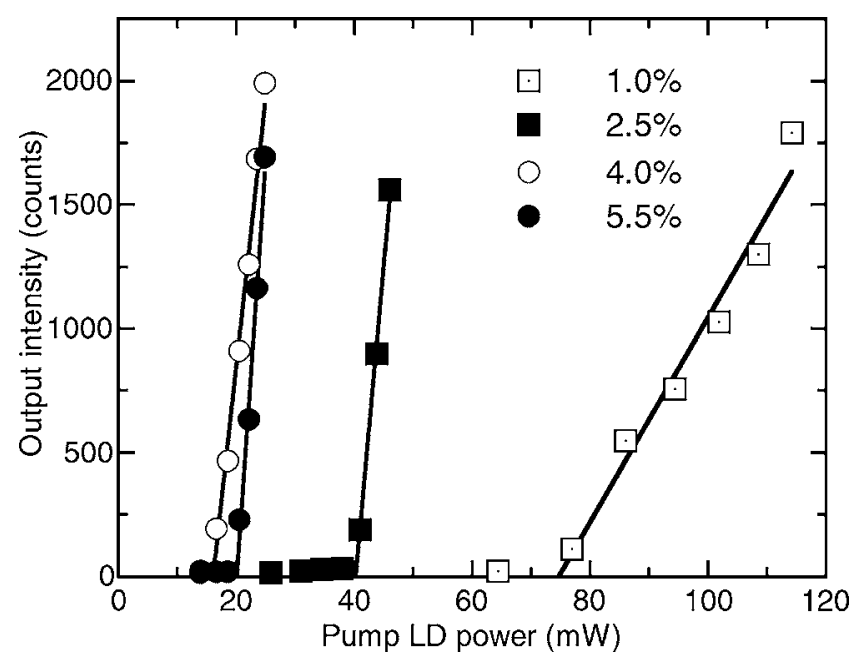

FIG. 4. Input-output characteristics of the DLE as a function of the peak power of the pump LD. The PVK films are doped with C540A concentrations of $1.0 \%$ (open squares), $2.5 \%$ (squares), $4.0 \%$ (open circles), and $5.5 \%$ (circles). 


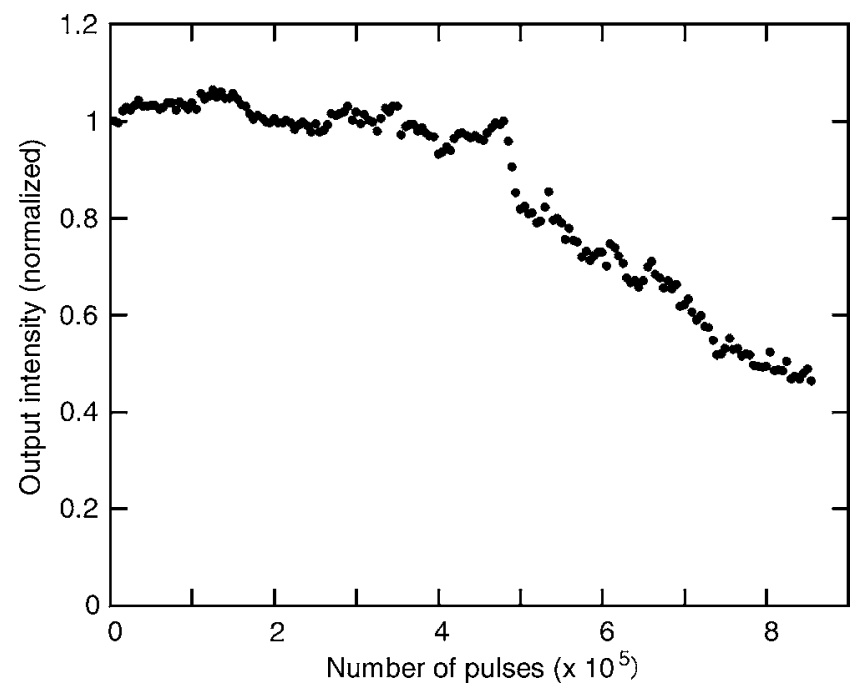

FIG. 5. Normalized output intensity as a function of the number of pump pulses.

reduced and the slope efficiency increased. The minimum lasing threshold was found to be $16 \mathrm{~mW} /$ pulse for a dye concentration of $4.0 \%$. In order to estimate the pump power absorbed by the active layer, we measured the transmittance and reflectance of the DLE at $441 \mathrm{~nm}$. The result revealed that $18.8 \%$ of the pump power was absorbed in the active layer; therefore, the absorbed pump power at threshold was estimated as $3 \mathrm{~mW} /$ pulse. By considering a pumping spot with a diameter of $\sim 20 \mu \mathrm{m}$, we estimated the densities of the threshold pump power and the absorbed threshold power as $5.1 \mathrm{~kW} / \mathrm{cm}^{2}$ and $960 \mathrm{~W} / \mathrm{cm}^{2}$, respectively. These values were comparable to those obtained for the guided-wave organic DFB lasers. ${ }^{4}$

The photostability of the active layer is vital when considering the practical applications of diode-pumped dye lasers. The operational lifetime of the DLE was measured by estimating the time taken for the emission intensity to decrease by a factor of 2 . Figure 5 shows the change in the output intensity of the DLE as a function of the number of pump pulse shots. The 4.0\%-doped DLE was irradiated with a LD pulse of $20 \mathrm{~mW}$ with a pulse duration of $3.3 \mathrm{~ns}$. After the emission of 800000 pulses at a rate of $1 \mathrm{kHz}$, the output intensity decreased to half of the value of the initial intensity. Previous studies have indicated that the degradation of dye molecules by the local heating of the active layer and by chemically active radicals causes a decrease in the emission intensity. ${ }^{8}$ The photostability of the polymeric dye laser can be improved by shortening the duration of the pump pulse to less than the order of nanoseconds and adding a singlet oxygen quencher into the active layer., ${ }^{2,9}$

In summary, we have demonstrated the performance of a diode-pumped polymeric dye laser that operates at around $550 \mathrm{~nm}$ with a threshold pump power of $16 \mathrm{~mW}$ by tuning the thickness and dye concentration of the active layer. It was found that the lifetime of the DLE can be extended to 800000 pulses when a blue LD with a 3.3 ns pulse is used as a pump source. The optimization of the mirror reflectivity and three-dimensional confinement of the laser cavity are currently under investigation in order to evaluate the output power and lasing efficiency.

${ }^{1}$ V. Bulović, V. G. Kozlov, V. B. Khalfin, and S. R. Forrest, Science 279, 553 (1998)

${ }^{2}$ Y. Oki, K. Aso, D. Zuo, N. J. Vasa, and M. Maeda, Jpn. J. Appl. Phys., Part 1 41, 6370 (2002).

${ }^{3}$ G. Heliotis, R. Xia, D. D. C. Bradley, G. A. Turnbull, I. D. W. Samuel, P. Andrew, and W. L. Barnes, Appl. Phys. Lett. 83, 2118 (2003).

${ }^{4}$ T. Riedl, T. Rabe, H.-H. Johannes, W. Kowalsky, J. Wang, T. Weimann, P. Hinze, B. Nehls, T. Farrell, and U. Scherf, Appl. Phys. Lett. 88, 241116 (2006).

${ }^{5}$ H. Sakata and H. Takeuchi, Electron. Lett. 43, 1431 (2007).

${ }^{6}$ S. S. Keskin, N. Aslan, and F. Bayrakçeken, Spectrochim. Acta, Part A 67, 1067 (2007).

${ }^{7}$ K. Yamashita, T. Kuro, K. Oe, and H. Yanagi, Appl. Phys. Lett. 88, 241110 (2006).

${ }^{8}$ A. Maslyukov, S. Sokolov, M. Kaivola, K. Nyholm, and S. Popov, Appl. Opt. 34, 1516 (1995).

${ }^{9}$ M. Ahmad, Opt. Commun. 271, 457 (2007). 